\title{
ESTRATÉGIAS PARA CONTROLE DE INFECCÇÃO HOSPITALAR CAUSADA POR ENTEROCOCCUS VANCOMICINA-RESISTENTES: UMA REVISÃO INTEGRATIVA
}

\section{STRATEGIES FOR THE CONTROL OF HOSPITAL INFECTIONS CAUSED BY VANCOMYCIN-RESISTANT ENTEROCOCCUS: AN INTEGRATIVE REVIEW}

\section{ESTRATEGIAS PARA CONTROLAR LA INFECCIÓN DEL HOSPITAL CAUSADA POR ENTEROCOCCUS RESISTENTE A LA VANCOMICINA: UNA REVISIÓN INTEGRATIVA}

Nathália Grosbelli Vicari ${ }^{1} \mathbb{\infty}$, Yago Marcos Pessoa Gonçalves ${ }^{2}$, Adriana Gonçalves de Oliveira ${ }^{3}$, Alessandra Barbosa Ferreira Machado ${ }^{4}$, Aline Dias Paiva ${ }^{5}$

\section{RESUMO}

Objetivo: avaliar a importância dos Enterococcus resistentes à Vancomicina (VRE) como agentes de infecções relacionadas à assistência à saúde (IRAS) e identificar as estratégias nacionais e internacionais usadas no controle de IRAS por VREs. Método: revisão integrativa da literatura. Seleção de 18 artigos que tratavam da importância de IRAS por VREs e das estratégias de controle. Resultados: observou-se concordância entre os artigos em relação às maneiras de minimizar os índices de IRAS por VREs. A ocorrência de IRAS é permissiva quando medidas para higiene são inadequadas. Ademais, treinamentos acerca do uso de antimicrobianos, culturas de vigilância e estruturação de uma comissão especializada são estratégias para redução de casos e que devem ser seguidas para contingência de VREs como agentes de IRAS. Conclusão: ações preventivas são a maneira mais eficiente de reduzir os casos de IRAS por VREs. É imprescindível padronizar e controlar o uso de antimicrobianos visando à prevenção da seleção de linhagens resistentes. Ainda, faz-se necessário manter ativas estratégias administrativas para controle de IRAS e atualização dos profissionais técnicos e da limpeza que atuam nos serviços de saúde, possibilitando educação continuada acerca de todos os tópicos que envolvam o controle dos casos.

Descritores: Controle de Infecção; Infecção Hospitalar; Assistência à Saúde; Resistência Bacteriana a Medicamentos; Enterococos Resistentes à Vancomicina.

\section{ABSTRACT}

Objective: to evaluate the importance of Vancomycin-resistant Enterococcus (VREs) as agents of Health care-associated infections (HAls) and to identify national and international strategies used in the control of HAls by VREs. Method: this is an integrative literature review. We selected 18 articles that dealt with the importance of HAls by VREs and the control strategies. Results: we noted an agreement among the articles regarding ways to minimize the rates of 
HAls by VREs. The occurrence of HAls is permissive when hygiene measures are inadequate. Furthermore, training on the use of antimicrobials, surveillance cultures, and the structuring of a specialized committee are strategies to reduce cases and should be followed for the contingency of VREs as agents of HAls. Conclusion: preventive actions are the most efficient way to reduce cases of HAls by VREs. It is essential to standardize and control the use of antimicrobials, in order to prevent the selection of resistant strains. Furthermore, it is necessary to keep active administrative strategies for the control of HAls and to update the technical and cleaning professionals who work in health services, enabling continuing education on all topics involving case control.

Descriptors: Infection Control; Cross Infection; Delivery of Health Care; Drug Resistance, Bacterial; Vancomycin-Resistant Enterococci.

\section{RESUMEN}

Objetivo: evaluar la importancia de los Enterococcus resistentes a la Vancomicina (VREs) como agentes de infecciones asociadas a la atención sanitaria (IAAS) e identificar las estrategias nacionales e internacionales utilizadas para controlar las IAAS causadas por los VREs. Método: revisión integradora de la literatura. Se seleccionaron 18 artículos que trataban sobre la importancia de las IAAS por los VREs y las estrategias de control. Resultados: se observó una concordancia entre los artículos sobre las formas de minimizar las tasas de IAAS por VREs. La aparición de IAAS es permisible cuando las medidas de higiene son inadecuadas. Además, los tratamientos sobre el uso de antimicrobianos, las culturas de vigilancia y la estructuración de una comisión especializada son estrategias para reducir los casos y que deben seguirse para la contingencia de los VREs como agentes de IAAS. Conclusión: las acciones preventivas son la forma más eficiente de reducir los casos de IAAS por VREs. Es imprescindible programar y controlar el uso de antimicrobianos para prevenir la aparición de líneas resistentes. Asimismo, es necesario mantener activas las estrategias administrativas para el control de las IAAS y la actualización de los profesionales técnicos y de limpieza que trabajan en los servicios sanitarios, permitiendo la formación continua en todos los temas que involucran el control de casos.

Descriptores: Control de infecciones; Infección Hospitalaria; Prestación de Atención de Salud; Farmacorresistencia Bacteriana; Enterococos Resistentes a la Vancomicina.

Descriptores: Control de infecciones; Infección Hospitalaria; Prestación de Atención de Salud; Farmacorresistencia Bacteriana; Enterococos Resistentes a la Vancomicina. 
1, 2, 3, 5 Universidade Federal do Triângulo Mineiro. Uberaba (MG), Brasil.

1๑https: / / orcid.org/0000-0001-7561-008X

2@https://orcid.org/0000-0002-9598-6276

3०https://orcid.org/0000-0003-4561-5503

$5 \odot$ https://orcid.org/0000-0003-4234-8892

${ }^{4}$ Universidade Federal de Juiz de Fora. Juiz de Fora (MG). Brasil.

4๑https://orcid..org/0000-0003-3972-7576

\section{Como citar este artigo}

Vicari NG, et al. Estratégias para controle de Infeccção Hospitalar causada por

Enterococcus Vancomicina-resistentes: uma revisão integrativa . Rev enferm UFPE on line. 2021;15:e247931 DOI: https://doi.org/10.5205/1981-8963.2021.247931

\section{INTRODUÇÃO}

A prevenção e o controle de infecções causadas por micro-organismos têm sido alvo de preocupação de estudiosos e profissionais da saúde desde épocas remotas. Ainda no século XIX, o médico húngaro Ignaz Semmelweis (1818-1865) já defendia a necessidade de procedimentos de antissepsia para o contato com os pacientes. ${ }^{1}$ Além dele, Joseph Lister (18271912), inspirado por estudos desenvolvidos por Louis Pasteur, também é reconhecido por seus feitos na antissepsia pré-cirúrgica. Entretanto, a prevenção da transmissão microbiana entre pessoas se torna mais relevante somente após a elucidação da relação entre micro-organismo e doença pelo médico alemão Robert Koch (1843-1910). ${ }^{2}$

Infecção é definida como a condição em que há reação tecidual e/ou fisiopatológica resultante da presença de micro-organismo infeccioso em um hospedeiro. ${ }^{3}$ As infecções hospitalares ou nosocomiais são hoje referenciadas como infecções relacionadas à assistência à saúde (IRAS). IRAS são aquelas que se manifestam em decorrência da hospitalização do paciente, das quais não havia evidência clínica no momento da internação, adquiridas no hospital por um paciente que foi internado por outra razão que não essa infecção. ${ }^{1,3}$

São exemplos de IRAS as infecções de sítio cirúrgico, pneumonias associadas à ventilação mecânica, infecções do trato urinário e da corrente sanguínea devido ao uso de cateter, vesical e venoso, respectivamente. De acordo com Fernando et al. $^{4}$, técnicas de higienização inadequadas ou insuficientes e o uso excessivo de antimicrobianos estão entre as principais causas de IRAS. O uso incorreto ou exagerado de antimicrobianos aumenta a pressão seletiva sobre comunidades microbianas, possibilitando a emergência de subpopulações de bactérias resistentes. ${ }^{1}$

A resistência microbiana é um tema de grande relevância no contexto da vigilância e monitoramento das IRAS, já que infecções causadas por bactérias resistentes a múltiplas clas- 
ses de antimicrobianos têm se tornado cada vez mais comuns, sendo de difícil tratamento e reduzindo as opções terapêuticas. Por isso, muitas vezes, esse tipo de IRAS está associado a um maior tempo de internação, maior risco de complicações, aumento da mortalidade e dos custos de hospitalização. ${ }^{5,6}$

Estudos sobre a prevalência de micro-organismos relacionados às IRAS destacam as bactérias Gram-negativas Pseudomonas aeruginosa, Acinetobacter, Klebsiella e Escherichia coli. Dentre as bactérias Gram-positivas, têm-se, principalmente, Staphylococcus aureus, Staphylococcus coagulase-negativos, Streptococcus pneumoniae e Enterococcus spp. .,4,7,8 $^{3}$ Atenção especial tem sido dada aos Staphylococcus aureus resistentes à Oxacilina/Meticilina (MRSA) e aos Enterococcus resistentes à Vancomicina (VREs). Entretanto, mais preocupante que a diversidade de agentes etiológicos é a emergência de linhagens resistentes, tornando este um importante problema de saúde pública. ${ }^{9}$

O gênero Enterococcus é composto por bactérias em forma de cocos, Gram-positivas, anaeróbias facultativas, capazes de sobreviver em condições ambientais desfavoráveis. ${ }^{10}$ Estão presentes em vasta gama de ambientes, incluindo o trato gastrointestinal de seres humanos e de outros animais, além de ser encontradas no solo, água, alimentos e superfícies. ${ }^{7,11}$ Entretanto, alguns representantes do gênero Enterococcus são capazes de causar infecções graves em pacientes imunocomprometidos ${ }^{8}$ ou naqueles submetidos à antibioticoterapia de largo espectro por tempo prolongado. ${ }^{7}$ Há espécies que são intrinsecamente resistentes à vancomicina, como Enterococcus gallinarum e Enterococcus casseliflavus. Todavia, a preocupação é em torno das espécies que apresentam resistência adquirida a esse antimicrobiano, como Enterococcus faecalis e Enterococcus faecium. ${ }^{10,12}$

A fim de controlar a disseminação de VRE, em 1995, o Centers for Disease Control and Prevention $(C D C)$, por meio do Comitê Consultivo para Práticas de Controle de Infecção Hospitalar, publicou um guia sobre o assunto. 0 guia recomendava educação continuada dos profissionais, higienização de mãos e de instrumentos, monitoramento, culturas de vigilância e restrição do uso de vancomicina como meios para diminuir o número de infecções e colonizações. ${ }^{13}$ Desde então, as instruções básicas fornecidas pelo CDC vêm sendo reforçadas como instrumentos de contenção da disseminação de VREs e de outras infecções causadas por bactérias resistentes. ${ }^{7,14}$

Considerando as expressivas consequências das IRAS causadas por VREs aos pacientes, profissionais da área da saúde e ao serviço hospitalar, esta revisão integrativa foi realizada com a finalidade de se obter respostas aos seguintes questionamentos: "Qual a importância 
dos VRES como agentes de IRAS e quais as principais ações propostas diante de IRAS causadas por VREs?".

\section{OBJETIVO}

Retratar a importância dos Enterococcus resistentes à Vancomicina (VRE) como agentes de infecções relacionadas à assistência à saúde (IRAS) e identificar as estratégias nacionais e internacionais usadas no controle e prevenção de IRAS por VREs.

\section{MÉTODO}

Trata-se de um estudo bibliográfico, tipo revisão integrativa de literatura, que seguiu etapas de elaboração de pergunta norteadora, estabelecimento de critérios de inclusão e exclusão, busca na literatura, análise crítica das informações e discussão dos resultados.

Com a proposta de analisar as IRAS causadas por VREs, realizou-se uma busca por artigos científicos nas bases de dados NCBI/PubMed e SciELO. Os trabalhos científicos foram pesquisados utilizando-se palavras-chave relacionadas à temática, a saber: "Enterococcus resistentes à Vancomicina”; “infecção hospitalar”; “controle de infecção hospitalar”; “estratégicas para prevenção de IRAS".

Os critérios de inclusão foram terem sido publicados entre 2015 e 2020, disponíveis na íntegra, nos idiomas português, inglês ou espanhol, com título e resumo coerentes com a intenção da pesquisa. Os critérios de exclusão abrangeram características que fugiam à intenção desta pesquisa ou aos critérios de inclusão.

O instrumento, elaborado com a finalidade de extrair e analisar os dados dos artigos selecionados, foi composto pelos seguintes itens: (1) 0 texto correlaciona a incidência de IRAS com a presença de microrganismos multirresistentes a antimicrobianos? (2) Qual a importância das infecções causadas por Enterococcus vancomicina-resistentes no ambiente hospitalar? (3) Quais as principais estratégias sugeridas para controle e acompanhamento de IRAS (discriminar elementos)? (4) Como devem atuar as comissões para controle de IRAS? (5) Como se dão as políticas estratégicas e a fiscalização no Brasil? (6) Qual a importância do controle do uso de antimicrobianos na prevenção de IRAS? (7) Como a restrição do contato com o paciente e as estratégias de educação profissional continuada impactam a ocorrência de IRAS por VREs?

\section{RESULTADOS}

Respeitando o fluxograma exposto abaixo (Figura 1), 18 artigos foram selecionados para a avaliação final (Tabela 1), além de documentos de órgãos oficiais, como ANVISA e CDC, importantes para a análise e descrição do assunto proposto. 


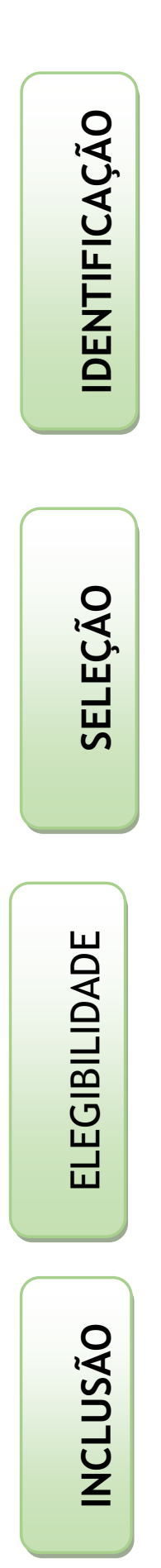

Registros identificados por meio de pesquisas nas bases de dados $(n=124)$

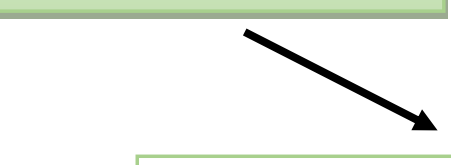

Registros identificados por meio de pesquisas em outras fontes de dados $(n=2)$

Registros após eliminação dos estudos duplicados

$(\mathrm{n}=98)$
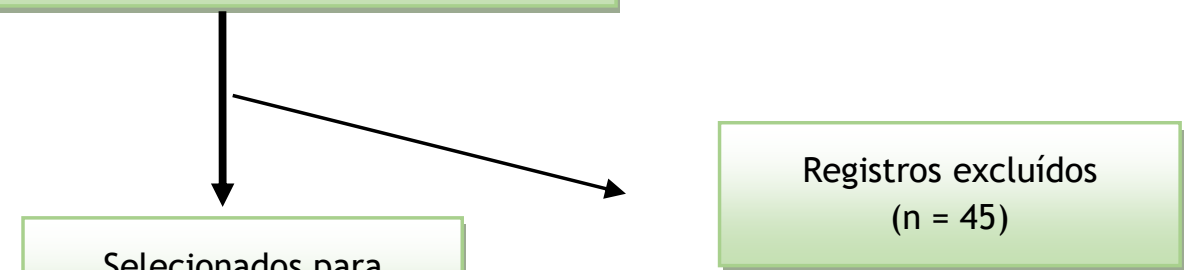

Selecionados para análise $(n=53)$

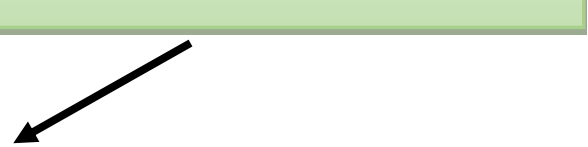

Trabalhos excluídos por não atenderem ao objetivo $(n=28)$

Artigos selecionados após

leitura íntegra

$(n=25)$

Estudos incluídos para análise e desenvolvimento do artigo $(n=18)$

Figura 1. Fluxograma da seleção dos estudos; adaptado do Preferred Reporting Items for Systematic Reviews and MetaAnalyses (PRISMA 2009). Uberaba, Brasil, 2020 
Core components for effective infection prevention and control programmes: new WHO evidence-based recommendations

Economic burden of nosocomial infections caused by vancomycin-resistant enterococci

Practical methods for effective vancomycin-resistant enterococci (VRE) surveillance: experience in a liver transplant surgical intensive care unit

The importance of adjusting for enterococcus species when assessing the burden of vancomycin resistance: a cohort study including over 1000 cases of enterococcal bloodstream infections

Continuous increase of vancomycin resistance in enterococci T causing nosocomial infections in Germany - 10 years of $\sum_{0}^{\circ}$

Transmission of Vancomycin-Resistant Enterococci in the Hospital Setting: Uncovering the Patient-Environment Interplay

Bloodstream infections caused by Enterococcus spp: A 10year retrospective analysis at a tertiary hospital in China

Healthcare-acquired infections: prevention strategies

O uso de antibióticos e as resistências bacterianas: breves notas sobre a sua evolução

Vancomycin-Resistant Enterococci: Epidemiology, Infection Prevention, and Control

Hospital-Associated Infections

Enterococcus spp. resistentes à vancomicina: carcaterísticas clínicas e fatores de risco

Perfil clínico e microbiológico dos casos de infecção hospitalar ocorridos em um hospital de médio porte do noroeste do Rio Grande do Sul
Babady NE

Raza T, Ullah SR, Mehmood K, Andleeb S

Perugini MRE, Yamada-Ogatta

Storr J, Twyman A, Zingg W, Antimicrob Resist Damani N, Kilpatrick C, Reilly J, Infect Control 2017 et al. Jan;6(1)

Puchter L, Chaberny IF, Antimicrob Resist InSchwab F, Vonberg RP, Bange FC, Ebadi E fect Control 2018 Jan;7(1)

Linfield RY, Campeau S, Injean Infect Control Hosp $P$, Gregson A, Kaldas F, Rubin Epidemiol 2018; 39(10)

$Z$, et al

Kramer TS, Remschmidt C, Antimicrob Resist InWerner S, Behnke M, Schwab fect Control 2018 Nov;7(1)

Remschmidt C, Schröder C, Antimicrob Resist Behnke $\mathrm{M}$, Gastmeier $\mathrm{P}$, Infect Control 2018 Apr;7(1)

Correa-Martinez CL, Tönnies Microorganisms 2020 $H$, Froböse NJ, Mellmann A, Jan;8(2) Kampmeier $\mathrm{S}$

Zheng Jx, Li H, Pu Zy, Wang Hy, J Huazhong Univ Sci Deng $X b$, Liu $X j$, et al Technolog Med Sci 2017;37(2)

Fernando AS, Grey TJ, Gottlieb Intern Med J 2017 Dez;47(12)

Loureiro RJ, Roque F, Teixeira Rev Port Sau Pub 2016 Jan;34(1)

Ramalheira

Reyes K, Bardossy AC, Zervos Infect Dis Clin North Am 2016 Dez; 30(4)

Microbiol Spectr 2016 Jun;4(3)

J Pak Med Assoc 2018 Mai;68(5)

Semina: Ciênc Biol SF, Pelisson M, Magalhães Saúde 2015 Ago; 36 GLG, Dias JB, Sugahara VH, et al.

Pozzato GS, Parisi MM Rev Bras Anal Clin 2018 Dez 16; 50(3) 
Enterococcus sp: Resistencia Antibacteriana en Infecciones Intrahospitalares
Rodríguez C, García S, Barberis Acta Bioquím Clín C, Saposnik E, Weyland B, Nastro $\mathrm{M}$, et al
Factores de riesgo de colonización por Enterococcus spp resistente a vancomicina en pacientes pediátricos hospitalizados con patología oncológica
Loyola P, Tordecilla J, Benadof Rev chil Infectol

D, Yohannessen K, Acuña M. 2015 Aug; 32(4)

Tabela 1. Artigos selecionados para análise e avaliação final. Elaborada pelos autores, Uberaba, Brasil, 2021

\section{IRAS e a resistência aos antimicrobianos}

O desenvolvimento de IRAS por um paciente está relacionado ao tempo de permanência no hospital, condição nutricional, utilização de antibióticos, natureza dos procedimentos e tratamentos realizados, grau de imunossupressão, realização de procedimentos invasivos e uso de cateteres e sondas. ${ }^{7,12,15} 0$ tempo de internação em unidades de tratamento intensivo (UTI), provavelmente pela maior necessidade de manuseio e cuidados por parte das equipes médicas e de enfermagem, aliado ao imunocomprometimento do paciente, está associado a maiores índices de IRAS. ${ }^{3}$ Além da UTI, os outros setores que costumam apresentar maior número de casos de IRAS são os de hemodiálise e oncologia. ${ }^{7}$

A transmissão de bactérias causadoras de IRAS ocorre (a) por meio de trabalhadores da área de saúde, incluindo residentes, médicos, enfermeiros e técnicos em enfermagem; (b) entre os pacientes; (c) pelos visitantes; (d) pelo ambiente e fômites. ${ }^{7,11,15}$

O ambiente hospitalar é um local de ampla utilização de antimicrobianos, tornando-se propício ao desenvolvimento de resistência bacteriana. O uso excessivo de antimicrobianos é descrito como um dos principais fatores que favorecem a emergência de linhagens resistentes em função da pressão seletiva. ${ }^{4,15}$

A pressão seletiva exercida pelo uso de antimicrobianos seleciona aquelas bactérias que, de alguma forma, modificaram seu material genético, possibilitando a sobrevivência na presença de um fármaco antes eficiente. Isso ocorre por mutações aleatórias no DNA ou por aquisição de novas sequências, a partir da transferência horizontal de elementos genéticos móveis, por exemplo. ${ }^{11}$ Essas modificações podem resultar em mecanismos de inativação da droga, alterações de vias enzimáticas, alterações no sítio de ação das drogas, bombas de eflu$\mathrm{xo}^{10}$, possibilitando o crescimento microbiano mesmo na presença do fármaco.

\section{IRAS causadas por Enterococcus vancomicina-resistentes (VREs)}


Os VREs começaram a surgir na década de 80, nos Estados Unidos (EUA) e na Europa, onde foi registrado o primeiro caso, em 1986. ${ }^{12}$ Nos EUA, o gênero Enterococcus é o segundo maior causador de IRAS, responsável por $14 \%$ dos casos, sendo o $E$. faecium resistente à vancomicina o agente responsável pela maioria das infecções de corrente sanguínea. Já na Europa, os índices de IRAS por VRE variam de 2 a $20 \% .^{12}$

No Brasil, o primeiro registro de infecção por VRE ocorreu em 1996. Atualmente, VRE tem sido considerado endêmico em hospitais brasileiros ${ }^{3,12}$, sendo responsável por surtos hospitalares de grande importância. ${ }^{12}$

Puchter et al. ${ }^{5}$, em um estudo caso-controle retrospectivo realizado na Alemanha, demonstraram que IRAS relacionadas a VREs elevam os custos hospitalares, principalmente quanto aos medicamentos e às despesas com equipe de enfermagem e produtos médicos. De acordo com a análise dos autores, os custos para tratamento de pacientes com infecções causadas por VREs são, em média, 13 mil euros superiores quando comparados aos custos com pacientes acometidos por infecções causadas por Enterococcus sensíveis.

As IRAS por VREs geralmente acometem o trato urinário e sítios estéreis, como o sangue, cuja cultura também apresenta ponta de cateter positiva ${ }^{12,16,17}$, sugerindo origem da infecção por meio da inserção do cateter. Infecção da corrente sanguínea causada por E. faecium resistente à vancomicina foi associada ao aumento do custo total da hospitalização e ao prolongamento da internação. ${ }^{18} \mathrm{O}$ número de casos e infecções de corrente sanguínea por VRE vem crescendo nos últimos anos, caracterizando uma ameaça à segurança dos pacientes. ${ }^{19}$

Ressalta-se ainda o elevado número de indivíduos colonizados pelo VRE, mas sem qualquer sinal indicativo da presença da bactéria. A colonização assintomática apresenta incidência 10 vezes maior quando comparada ao processo patológico ${ }^{7}$, sendo uma condição descrita como mais permissiva a falhas na higienização e consequente transmissão da bactéria.

\section{Estratégias de controle e acompanhamento das IRAS}

As taxas de infecções causadas por bactérias do gênero Enterococcus vêm crescendo ao longo dos anos. Segundo Remschmidt et al. ${ }^{19}$, na Alemanha, as IRAS por Enterococcus passaram de 1,4\% em 2007 para mais de 10\% em 2016.

A prioridade na administração dos casos de colonização ou infecção é o controle das fontes. ${ }^{7}$ Pela capacidade de disseminação de bactérias do gênero Enterococcus e pelo seu perfil de endemicidade, se o controle das fontes não for realizado no início e não tiver aplicação constante, as tentativas posteriores para erradicação do problema se tornam quase impossíveis. $^{12}$ 
Estudos nacionais e internacionais revelam que as práticas de higienização adequada aplicadas às mãos, roupas de cama, superfícies e equipamentos, aliadas às precauções de contato entre pacientes, visitas e equipe de enfermagem, com a rotatividade e restrição no uso de antimicrobianos, são ações recomendadas para prevenção ou diminuição da ocorrência de IRAS por VREs. ${ }^{4,7-9,15}$ Ainda, o controle das IRAS pode ser otimizado com educação continuada dos profissionais ${ }^{4}$ e com a manutenção de comissões e políticas de controle hospitalar. ${ }^{17}$

Além das ações supracitadas, o acompanhamento dos casos de colonização ou de infecção pode ser feito por culturas de vigilância, ${ }^{4,15}$ realizadas periodicamente e em pacientes assintomáticos, sendo uma maneira de fazer o screening da colonização por VRE. 7,12

\subsection{Higienização das mãos}

As mãos são consideradas importantes vias de transmissão de VRE entre pacientes ${ }^{7}$ e, por esse motivo, a prática de higienização de mãos é considerada a melhor maneira de demonstrar a qualidade em serviços de saúde e prevenir IRAS. 4,17,20 Ela deve ser realizada antes e depois do contato com pacientes ou com o ambiente em que os pacientes se encontram. ${ }^{4,12,21}$

Uma boa higiene das mãos é o princípio fundamental da prevenção de IRAS por VRE, além de ser um indicativo de segurança nos hospitais ${ }^{4}$. 0 monitoramento da higienização das mãos nos serviços de saúde, por meio de um esquema que garanta um feedback para os profissionais, deve ser feito como uma forma de demonstrar a performance dos mesmos no controle e prevenção de IRAS ${ }^{22}$, permitindo mudanças comportamentais acerca das práticas de prevenção.

Quando eficiente, a higienização das mãos é capaz de reduzir em $47 \%$ os casos de infecção por VRE. ${ }^{7}$ A prática realizada de maneira adequada compreende o uso de água e sabão, mantendo a fricção das mãos por, no mínimo, 30 segundos, o que permite a eliminação dos VREs presentes. ${ }^{7}$ Mãos que foram higienizadas por 5 segundos não mostraram impacto algum no conteúdo microbiano ${ }^{7,11}$ e podem continuar transmitindo VREs.

O acesso a lavatórios deve ser prontamente disponibilizado em locais de atenção à saúde e em quantidade e distribuição que permitam sua utilização frequente. ${ }^{22}$ Os serviços de saúde devem se adequar às recomendações da Organização Mundial da Saúde, no que se refere ao número e à posição mais adequada para equipamentos de higiene das mãos, garantindo que essa prática não deixe de ser realizada por dificuldade de acesso à água, sabão e álcool. ${ }^{22}$

\subsection{Higienização de superfícies e equipamentos}

Assim como as mãos, fômites podem ser disseminadores de VREs. Portanto, sua higienização deve ser considerada como componente-chave nos programas de controle de IRAS ${ }^{4}$, uma vez que os VREs podem sobreviver em superfícies secas e equipamentos por meses..$^{4,7}$ 
Reyes et al. ${ }^{7}$ recomendam que os serviços de saúde sigam alguns protocolos de limpeza e que estes sejam realizados com frequência ininterrupta. Contudo, o monitoramento da eficiência dos protocolos pode ser periódico e deve abranger a realização de culturas com amostras de superfícies e equipamentos, uma vez que os VREs podem sobreviver no ambiente por até um ano.

Dentre as maneiras de realizar a descontaminação desses locais, a mais comum é o uso de hipoclorito de sódio. Contudo, há outras formas que apesar de terem custo mais elevado não demandam ação direta de profissionais de limpeza, como é o caso de iluminação ultravioleta (UV) e vaporização com peróxido de hidrogênio (VPH). ${ }^{4}$

De acordo com estudo realizado por Eckstein et al. ${ }^{23}$, a limpeza tradicional demonstra uma eficiência de $29 \%$ na prevenção da transmissão de VREs, uma vez que, após higienização dos quartos onde haviam pacientes colonizados por VREs, 71\% das amostras ambientais ainda se mostraram positivas. ${ }^{7,23}$ Todavia, outro estudo mostrou que pacientes admitidos em quartos que passaram por VPH têm 80\% menos chances de adquirir VRE e quando a desinfecção é feita por UV as chances são $31 \%$ menores. ${ }^{4}$

No hospital universitário de Münster, na Alemanha, Correa-Martínez e colaboradores ${ }^{15}$ compararam filogeneticamente linhagens de VREs identificadas em culturas do ambiente hospitalar, realizadas periodicamente como parte do controle de IRAS do hospital, e linhagens de VREs de culturas de vigilância a partir de swab retal, feitas como screening de rotina nos pacientes hospitalizados. Segundo os autores, havia semelhança genética entre as linhagens, confirmando que o ambiente hospitalar atua como reservatório, facilitando a expansão dos casos de IRAS por VREs. Nesse mesmo estudo, observou-se que casos de IRAS relacionados a uma mesma linhagem de VRE poderiam ser postergados devido à sobrevivência dos VREs no ambiente hospitalar ${ }^{15}$, ou seja, uma linhagem que estava causando infecção em dado período pode permanecer no ambiente por muito tempo, em superfícies e equipamentos, causando infecções futuras em novos pacientes.

\subsection{Culturas de vigilância}

O uso de culturas de vigilância é considerado útil para detecção e intervenção preco$\mathrm{ce}^{4,8}$ e tem se mostrado benéfico na redução do número de infecções por Enterococcus e também na redução da transmissão bacteriana entre pacientes de alto risco. ${ }^{7}$

Culturas de vigilância são realizadas por meio de amostras coletadas com swab retal ou perianal ou também por meio de coprocultura. ${ }^{7}$ As amostras são cultivadas em meios sólidos, que podem ser de diferentes tipos, com destaque para os VRE-seletivos e cromogênios. ${ }^{8,15} \mathrm{Em}$ situações de surto, deve-se considerar ainda a coleta de amostras de feridas e de pele. ${ }^{7}$ 
Após resultados positivos na cultura, é importante a realização do teste de suscetibilidade a antimicrobianos (TSA) para avaliação do perfil de sensibilidade da linhagem isolada. ${ }^{4}$ Enterococcus são considerados resistentes quando a Concentração Inibitória Mínima (CIM) de vancomicina é maior ou igual a $32 \mu \mathrm{g} / \mathrm{mL}^{7}$

A implementação de sistemas de vigilância utilizando-se screening com swabs perianais se torna mais confiável quando há sistemas que permitem identificação precoce de pacientes em risco. ${ }^{4}$ De acordo com Reyes et al. ${ }^{7}$, culturas de vigilância são de grande importância, principalmente em populações específicas, como pacientes em diálise, na UTI, hospitalizados por longos períodos, oncológicos e transplantados.

Por outro lado, Linfield e colaboradores ${ }^{24}$, em estudo realizado na UTI do centro médico Ronald Reagan UCLA, na Califórnia, observaram que a amostragem utilizando-se swab perianal foi mais sensivel ( $\mathrm{S}=96 \%$ ) quando comparada à coprocultura $(\mathrm{S}=84 \%)$ na identificação de VRE. Segundo os mesmos autores, $63 \%$ dos pacientes não estavam colonizados com VRE no momento da admissão, assim como 39,1\% dos que haviam sido admitidos antes do estudo. Esses pacientes, considerados casos negativos, foram avaliados semanalmente e $25 \%$ deles foram contaminados de alguma forma com linhagens VREs. Essa avaliação demonstra mais uma vez a importância de culturas de vigilância em pacientes hospitalizados.

\section{Comissões para Controle de IRAS}

A formação e apoio de grupos especializados, constituídos por profissionais capacitados, é uma prática vantajosa no processo de contenção de casos de IRAS ${ }^{21}$. Programas de controle guiados por esses grupos facilitam a implementação de ações estratégicas nos serviços de saúde. ${ }^{4}$

Segundo Storr e colaboradores ${ }^{22}$, dentre os componentes-chave para prevenção e controle de IRAS, destacam-se, em primeiro lugar, os programas geridos por comissões especializadas. Essas comissões devem ser compostas por profissionais treinados, de distintas áreas de formação ${ }^{25}$, e em número suficiente, sendo 1 para cada 100 ou 250 leitos, dependendo da situação do serviço de saúde. ${ }^{22}$

No Brasil, durante a década de 60 , criou-se a primeira Comissão de Controle de Infecção hospitalar $(\mathrm{CCIH})$ e cerca de 20 anos depois surgia a Associação Brasileira de Profissionais em Controle de Infecção Hospitalar (ABIH). ${ }^{26}$ Com a consolidação da ABIH, normas e regulamentações foram propostas, diminuindo, assim, os casos de IRAS em todo o país. ${ }^{26}$

\section{Políticas Estratégicas e Fiscalização no Brasil}

Em novembro de 2016, foi publicado pela ANVISA o Programa Nacional de Prevenção e Controle de Infecções Relacionadas à Assistência à Saúde (PNPCIRAS). Trata-se de um docu- 
mento que reúne políticas e programas voltados à redução dos casos de IRAS no Brasil, contendo objetivos e metas a serem alcançados pelos serviços de saúde entre os anos de 2016 a 2020. ${ }^{21}$ Estabeleceram-se como objetivos específicos consolidar o sistema nacional de vigilância epidemiológica das IRAS; reduzir nacionalmente a incidência das IRAS prioritárias; prevenir e controlar a disseminação da resistência microbiana em serviços de saúde; e consolidar o PNPCIRAS. ${ }^{21}$

Para cada um dos objetivos a serem alcançados há uma tabela com a descrição de atividades que devem ser seguidas pelos serviços de saúde. As atividades, em geral, visam ao fortalecimento de CCIHs e de programas estaduais e nacionais de prevenção de IRAS, à elaboração de métodos avaliativos para a eficiência das ações, à qualificação de profissionais que atuam diretamente com os pacientes e dos laboratórios de diagnóstico microbiológico. ${ }^{21,25}$

A importância de manter programas de nível nacional, como o PNPCIRAS, é defendida por Gomes e Moraes $^{27}$, que também ressaltam a relevância em se manter a fiscalização. Segundo os autores, é competência da ANVISA a interdição de estabelecimentos que violem regras de biossegurança, além da averiguação do cumprimento de normas sanitárias que envolvam segurança do paciente e prevenção de IRAS. Deve ainda haver fiscalização da ação das $\mathrm{CClHs}$, bem como cooperação técnica quando demandada. ${ }^{27}$

Contudo, falhas nos atos de fiscalização e auxílio dos estabelecimentos de saúde na implementação de instrumentos de combate ao avanço e diminuição da incidência de IRAS têm sido reportadas. ${ }^{25,27}$

\section{Restrições e Controle do Uso de Antimicrobianos}

No ambiente hospitalar ocorre o uso intenso e frequente de diversos tipos de antimicrobianos. Esse fato é entendido como um mecanismo que propicia a seleção de linhagens bacterianas resistentes. ${ }^{7,27}$ Diversos estudos relacionam o uso de antimicrobianos com casos de IRAS por micro-organismos multirresistentes. ${ }^{4,9,11,12,17,26}$

A otimização do uso de antimicrobianos, incluindo dose, tempo e via de administração, é uma estratégia-chave na prevenção de IRAS. ${ }^{4}$ Nesse contexto, o PNPCIRAS visa à publicação de uma diretriz nacional para tratar do uso controlado de antimicrobianos como parte das estratégias focadas no treinamento de profissionais. ${ }^{21}$

Estratégias de restrição, combinadas com educação acerca da prescrição, são consideradas fundamentais na prevenção de seleção de micro-organismos resistentes. ${ }^{4,22} \mathrm{~A}$ elaboração de protocolos sobre a utilização dos antimicrobianos também é entendida como uma estratégia para adequar sua utilização no ambiente hospitalar ${ }^{21,26}$ de forma a padronizar a escolha 
das classes corretas, uma vez que até $50 \%$ das prescrições são, de alguma maneira, inadequadas. $^{4}$

\section{Precauções de contato}

O contato, seja direto pessoa-pessoa, com fluidos ou fômites, é entendido como umas das principais causas de transmissão de micro-organismos, levando a um possível aumento na incidência de IRAS. Precauções de contato são recomendadas para as infecções transmitidas por meio de contato direto com pacientes infectados ou colonizados, ou com fômites ${ }^{8}$, e quando se trata do cuidado ao paciente hospitalizado ou da manipulação de fluidos corporais. ${ }^{21,27}$

Não há estudos de intervenção que tenham comparado as taxas de aquisição de VRE com precauções de contato versus precauções padrão realizadas na rotina. ${ }^{4}$ Contudo, ações e políticas de precauções de contato com pacientes devem ser realizadas quando se tratam de casos de surtos de IRAS. ${ }^{7}$ Além do uso de equipamentos de proteção individual, como luvas, jalecos, máscaras e óculos, quando necessário, pode-se alojar pacientes em quartos individuais, de modo a restringir o contato. ${ }^{4}$

A não ocupação do hospital além dos limites preestabelecidos, como o número máximo de leitos por quarto, é uma ação básica ${ }^{22}$ a ser seguida a fim de dificultar a ocorrência de casos de IRAS por VRE, bem como por outros micro-organismos.

\section{Educação Continuada}

Ações educativas são compreendidas como estratégias primárias para a solução do problema das IRAS ${ }^{25}$ e estão entre as recomendações dos programas de controle de IRAS no Brasil, Como PNPCIRAS. ${ }^{21}$ A educação acerca da colonização por VRE deve ser realizada desde o primeiro dia de hospitalização ${ }^{20}$, já que é entendida como uma atividade preventiva. Além das atividades educativas, avaliações periódicas da efetividade dos treinamentos também precisam ser realizadas. ${ }^{22}$

As atividades educativas devem abranger todas as demais estratégias para controle de IRAS. ${ }^{4,7}$ Higienização de mãos, equipamentos e ambientes, interpretação de protocolos, controle do uso de antimicrobianos e precauções de contato precisam ser pautas da educação continuada, uma vez que a capacitação dos profissionais é o que sustenta a adoção de práticas adequadas. ${ }^{25}$

\section{DISCUSSÃO}

Elencaram-se em 10 dos artigos analisados $3,5,7,11,12,15,16,17,18,19$ os Enterococcus vancomicina-resistentes como principais causas de IRAS nos últimos anos. A morbidade e a mortalidade 
associadas aos casos de IRAS por VREs, com o aumento dos gastos hospitalares para tratamento dos pacientes, reforçam a preocupação com colonizações e infecções por VREs.

Percebeu-se, quanto à organização temática, que seis artigos $4,7,12,17,20,22$ apontaram a higienização das mãos como uma das estratégias mais importantes para o controle de IRAS, independente do agente etiológico, enquanto cinco artigos defendem o monitoramento por meio de culturas de vigilância como estratégia fundamental no controle da incidência de IRAS em diferentes hospitais. Em três artigos, estabeleceram-se ligações concretas entre superfícies contaminadas e a colonização de pacientes por VREs. ${ }^{4,7,15}$ Confirma-se, assim, que as mãos ainda continuam sendo as principais vias de transmissão de patógenos, incluindo VREs, mas evidencia-se também o papel de superfícies inertes na dinâmica de disseminação desses patógenos, principalmente considerando a capacidade de sobrevivência dos enterococos a condições ambientais adversas.

Constatou-se que três artigos ${ }^{4,22,26}$ enfatizam a importância da atuação de Comissões de Controle de Infecção Hospitalar com vistas à redução de casos de IRAS. A formação de equipes com profissionais capacitados é capaz de facilitar a implementação de ações estratégicas nos serviços de saúde, o que vai de encontro ao recomendado pelo PNPCIRAS, da ANVISA.

Identificou-se que somente um artigo ${ }^{27}$ discute a função de fiscalização da Agência Nacional de Vigilância Sanitária nos casos de infecção hospitalar. Os autores apontam como fundamentais a fiscalização e a aplicação correta do PNPCIRAS.

Identificou-se que a restrição e o controle do uso de antimicrobianos no ambiente hospitalar são considerados em nove artigos $4,7,9,11,12,17,22,26,27$ como uma estratégia essencial para a prevenção de IRAS. A otimização do uso de antimicrobianos incluiria a adequação da prescrição (escolha correta do fármaco), ajuste de dose, tempo e via de administração, com a elaboração de protocolos a serem seguidos no ambiente hospitalar.

Demonstrou-se em quatro dos artigos ${ }^{4,8,22,27}$ que a restrição do contato com o paciente pode reduzir a disseminação de patógenos no ambiente hospitalar. As estratégias de educação profissional continuada também foram associadas a uma redução da ocorrência de IRAS por VREs $4,7,20,22$.

Enfatiza-se que as ações de controle e prevenção de IRAS devem ser baseadas na especificidade do sistema de saúde, ou seja, devem ser direcionadas aos micro-organismos causadores das IRAS nos respectivos locais. Naqueles locais em que o número de casos de IRAS por VREs se mostra elevado, o monitoramento da colonização do paciente por VRE deve ser mantido como atividade de rotina do serviço. A educação profissional continuada deve ser vista 
como atividade preventiva e essencial, devendo ser estimulada entre os diferentes setores hospitalares.

Contatou-se como limitação as escassas evidências científicas robustas sobre as estratégias específicas e particularidades a serem adotadas para controle de IRAs por VREs. Acredita-se que tal condição seja devida, em parte, ao fato das bactérias Gram-negativas terem sido, durante muito tempo, os principais agentes de IRAS e, consequentemente, o foco das estratégias de controle. Entretanto, bactérias Gram-positivas têm emergido nos últimos anos como causas importantes de IRAS, não só em hospitais brasileiros, mas também em âmbito internacional, tornando primordial o controle desses patógenos para se obter resultados positivos e eficazes de controle de infecções no ambiente hospitalar.

\section{CONCLUSÃO}

A preocupação com o controle de IRAS é tão antiga quanto sua descoberta, mas apesar disso parece que a implementação de estratégias de controle microbiano adotadas em ambiente hospitalar ainda é insuficiente para erradicar esse problema tão comum e ao mesmo tempo tão grave, que além de prejuízos à saúde traz prejuízos econômicos.

Com todo o conhecimento acerca da emergência de resistência bacteriana e da incidência de IRAS, como aquelas causadas por VRE, fica clara a necessidade de manter práticas de higienização eficientes nos hospitais e serviços de saúde, compreendida internacionalmente como ação base para redução nos casos de IRAS. O preparo para tais ações deve incluir desde os profissionais de saúde até os funcionários da limpeza.

As culturas de vigilância permitem detecção e intervenção precoces, e esse monitoramento dos casos deve ser realizado com frequência e periodicidade apropriadas. Além disso, faz-se necessário desenvolver estratégias que adequem o uso de antimicrobianos, possibilitando educação continuada aos profissionais que os prescrevem e administram.

A educação continuada acerca das intervenções realizadas para reduzir as IRAS é outro fato que está em consonância com estudos nacionais e internacionais, devendo, portanto, ser realizada de forma abrangente com todos os profissionais compreendidos na atenção hospitalar e tratando dos diversos temas que envolvam minimização de IRAS. Todos os processos devem ser acompanhados por um comitê ou uma equipe técnica focada no controle de IRAS a fim de garantir a segurança dos pacientes hospitalizados.

Defende-se, dessa forma, que este estudo apresenta relevância para o ensino, a pesquisa e a prática clínica, uma vez que busca o conhecimento da importância dos Enterococcus vancomicina-resistentes como agentes de IRAS e das estratégias a serem adotadas para mini- 
mizar sua ocorrência no ambiente hospitalar. As discussões sobre a temática são pertinentes e poderão contribuir para auxiliar a equipe multiprofissional no controle desses patógenos, contribuindo, assim, para a melhoria da qualidade do tratamento oferecido ao paciente hospitalizado, priorizando a eficácia da assistência prestada.

\section{CONTRIBUIÇÕES}

Nathália Grosbelli Vicari. Elaboração do manuscrito.

Yago Marcos Pessoa Gonçalves. Elaboração do manuscrito.

Adriana Gonçalves de Oliveira. Correção e análise do manuscrito.

Alessandra Barbosa Ferreira Machado. Correção e análise do manuscrito.

Aline Dias Paiva. Elaboração, correção e análise do manuscrito.

\section{CONFLITO DE INTERESSES}

Nada a declarar.

\section{REFERÊNCIAS}

1. Santos NQ. A resistência bacteriana no contexto da infecção hospitalar. Texto Contexto Enferm.2004 Jan;13(1):64-81. Disponível em:

https: / / www.scielo.br/scielo.php?pid=S010407072004000500007\&script=sci_arttext\&tlng $=\mathrm{pt}$

2. Bencko V, Schejbalová M. From Ignaz Semmelweis to the Present: Crucial Problems of Hospital Hygiene. Indoor Built Environ 2006 Fev;15(1):3-7.

https://www.researchgate.net/publication/247731782_From_lgnaz_Semmelweis_to_the Present_Crucial_Problems_of_Hospital_Hygiene

3. Pozzato GS, Parisi MM. Perfil clínico e microbiológico dos casos de infecção hospitalar ocorridos em um hospital de médio porte do noroeste do Rio Grande do Sul. Rev Bras Anal Clin 2018 Dez 16;50(3):260-264. http://www.rbac.org.br/artigos/perfil-clinico-emicrobiologico-dos-casos-de-infeccao-hospitalar-ocorridos-em-um-hospital-de-medioporte-do-noroeste-do-rio-grande-do-sul//

4. Fernando AS, Grey TJ, Gottlieb T. Healthcare-acquired infections: prevention strategies. Intern Med J 2017 Dez;47(12):1341-1351.

https://onlinelibrary.wiley.com/doi/full/10.1111/imj.13642

5. Puchter L, Chaberny IF, Schwab F, Vonberg RP, Bange FC, Ebadi E. Economic burden of nosocomial infections caused by vancomycin-resistant enterococci. Antimicrob Resist In- 
fect Control 2018 Jan;7(1):1-7.

https://www.ncbi.nlm.nih.gov/pmc/articles/PMC5755438/

6. Tacconelli E, Cataldo MA. Vancomycin-resistant enterococci (VRE): transmission and control. Int J Antimicrob Agents 2008 Fev;31(2): 99-106.

https: / /pubmed.ncbi.nlm.nih.gov/18164908/

7. Reyes K, Bardossy AC, Zervos M. Vancomycin-Resistant Enterococci: Epidemiology, Infection Prevention, and Control. Infect Dis Clin North Am 2016 Dez;30(4):953-

965. https://pubmed.ncbi.nlm.nih.gov/27660091/

8. Babady NE. Hospital-Associated Infections. Microbiol Spectr 2016 Jun;4(3):1-22.

https://www.asmscience.org/content/journal/microbiolspec/10.1128/microbiolspec.DM $\underline{\mathrm{IH} 2-0003-2015}$

9. Loureiro RJ, Roque F, Teixeira Rodrigues A, Herdeiro MT, Ramalheira E. O uso de antibióticos e as resistências bacterianas: breves notas sobre a sua evolução. Rev Port Sau Pub 2016 Jan;34(1):77-84.

https://www.sciencedirect.com/science/article/pii/S087090251500067X

10. Murray PR, Rosenthal K, Pfaller MA. Microbiologia Médica. 6th ed. e atual. Rio de Janeiro: Elsevier; 2009. 946 p. ISBN: 9788535234466.

11. Raza T, Ullah SR, Mehmood K, Andleeb S. Vancomycin resistant Enterococci: A brief review. J Pak Med Assoc 2018 Mai;68(5):768-772.

https://pubmed.ncbi.nlm.nih.gov/29885179/

12. Perugini MRE, Yamada-Ogatta SF, Pelisson M, Magalhães GLG, Dias JB, Sugahara VH, et al. Enterococcus spp. resistentes à vancomicina: características clínicas e fatores de risco. Semina: Ciênc Biol Saúde 2015 Ago;36(1):291-301.

http://www.uel.br/revistas/uel/index.php/seminabio/article/view/19393/16977

13. CDC. Centers For Disease Control And Prevention [Internet]. Atlanta; 1995 sep. Recommendations for Preventing the Spread of Vancomycin Resistance; [Acesso em $01 \mathrm{abr}$ 2020]; Disponível em: https://www.cdc.gov/mmwr/preview/mmwrhtml/00039349.html

14. Weinstein RA. Controlling antimicrobial resistance in hospitals: infection control and use of antibiotics. Emerg Infect Dis 2001;7(2):188-192.

https://www.ncbi.nlm.nih.gov/pmc/articles/PMC2631704/

15. orrea-Martinez CL, Tönnies H, Froböse NJ, Mellmann A, Kampmeier S. Transmission of Vancomycin-Resistant Enterococci in the Hospital Setting: Uncovering the PatientEnvironment Interplay. Microorganisms 2020 Jan;8(2):203-210. 
16. Zheng Jx, Li H, Pu Zy, Wang Hy, Deng Xb, Liu Xj, et al. Bloodstream infections caused by Enterococcus spp: A 10-year retrospective analysis at a tertiary hospital in China. $J$ Huazhong Univ Sci Technolog Med Sci 2017;37(2):257-263. https://pubmed.ncbi.nlm.nih.gov/28397040/

17. Rodríguez C, García S, Barberis C, Saposnik E, Weyland B, Nastro M, et al. Enterococcus sp: Resistencia Antibacteriana en Infecciones Intrahospitalares. Acta Bioquím Clín Latinoam 2015;47(1):155-160. https://pesquisa.bvsalud.org/portal/resource/pt/lil-727414

18. Kramer TS, Remschmidt C, Werner S, Behnke M, Schwab F, Werner G, et al. The importance of adjusting for enterococcus species when assessing the burden of vancomycin resistance: a cohort study including over 1000 cases of enterococcal bloodstream infections. Antimicrob Resist Infect Control 2018 Nov;7(1):1-9.

https://pubmed.ncbi.nlm.nih.gov/30459945/

19. emschmidt C, Schröder C, Behnke M, Gastmeier P, Geffers C, Kramer TS. Continuous increase of vancomycin resistance in enterococci causing nosocomial infections in Germany - 10 years of surveillance. Antimicrob Resist Infect Control 2018 Apr;7(1):1-7. https://www.ncbi.nlm.nih.gov/pmc/articles/PMC5937822/

20. Loyola P, Tordecilla J, Benadof D, Yohannessen K, Acuña M. Factores de riesgo de colonización por Enterococcus spp resistente a vancomicina en pacientes pediátricos hospitalizados con patología oncológica. Rev chil Infectol 2015 Aug;32(4): 393-398. https://scielo.conicyt.cl/scielo.php?script=sci_arttext\&pid=S0716-10182015000500004

21. NVISA: Agência Nacional De Vigilância Sanitária [Internet]. 2016. Programa Nacional De Prevenção E Controle De Infecções Relacionadas à Assistência à Saúde (2016-2020). [Acesso em 6 mai 2020]; Disponível em:

https://www20.anvisa.gov.br/segurancadopaciente/index.php/publicacoes/item/pnpcir as-2016-2020

22. torr J, Twyman A, Zingg W, Damani N, Kilpatrick C, Reilly J, et al. Core components for effective infection prevention and control programmes: new WHO evidence-based recommendations. Antimicrob Resist Infect Control 2017 Jan;6(1):1-18.

https://pubmed.ncbi.nlm.nih.gov/28078082/

23. Eckstein BC, Adams DA, Eckstein EC, Rao A, Sethi AK, Yadavalli GK, et al. Reduction of Clostridium Difficile and vancomycin-resistant Enterococcus contamination of environmental surfaces after an intervention to improve cleaning methods. BMC Infect Dis 2007 June;7(1):1-6. https://www.ncbi.nlm.nih.gov/pmc/articles/PMC1906786/ 
24. Linfield RY, Campeau S, Injean P, Gregson A, Kaldas F, Rubin Z, et al. Practical methods for effective vancomycin-resistant enterococci (VRE) surveillance: experience in a liver transplant surgical intensive care unit. Infect Control Hosp Epidemiol 2018;

39(10):1178-1182. https://pubmed.ncbi.nlm.nih.gov/30178725/

25. Nogueira Junior C. Políticas públicas para prevenção e controle de IRAS: concepção de um modelo explicativo para sua estruturação [Dissertação]. São Paulo: Escola de Enfermagem da Universidade de São Paulo (USP); 2018. Mestrado em Enfermagem.

https://www.teses.usp.br/teses/disponiveis/7/7141/tde-22112018-162054/pt-br.php

26. Padoveze MC, Melo S, Bishop S, Poveda VB, Fortaleza, CMCB. Public policies on healthcare-associated infections: a Brazil and UK case study. Rev Saude Publica 2017 dec;51(119):1-7. https: //www.scielo.br/scielo.php?pid=S0034$\underline{89102017000100608 \& \text { script }=\text { sci_abstract\&tlng=en }}$

27. Gomes $M$, Moraes V. $O$ programa de controle de infecção relacionada à assistência à saúde em meio ambiente hospitalar e o dever de fiscalização da Agência Nacional de Vigilância Sanitária. Rev Direito Sanit 2018 mar;18(3):43-61. http://www.revistas.usp.br/rdisan/article/view/144647

\section{Correspondência}

Aline Dias Paiva

E-mail: alinedpaiva@yahoo.com.br

Submissão: 19/08/2020

Aceito: 19/04/2021

Copyright $\odot 2021$ Revista de Enfermagem UFPE on line.

(c) Er Este é um artigo de acesso aberto distribuído sob a Atribuição CC BY 4.0 Creative Commons AttributionShareAlike 4.0 International License, a qual permite que outros distribuam, remixem, adaptem e criem a partir do seu trabalho, mesmo para fins comerciais, desde que lhe atribuam o devido crédito pela criação original. É recomendada para maximizar a disseminação e uso dos materiais licenciados. 